INSTITUTO EDUCACIONAL WILSON WANDERLEY

PÓS-GRADUAÇÃO EM PROCEDIMENTOS INTRADÉRMICOS E SUBCUTÂNEOS

\author{
LUCIMARA SARGIANI FABOSSI
}

ESTRATÉGIAS TERAPÊUTICAS DO ESTETICISTA FRENTE AO PACIENTE PORTADOR DE HIDRADENITE SUPURATIVA 


\section{LUCIMARA SARGIANI FABOSSI}

\section{ESTRATÉGIAS TERAPÊUTICAS DO ESTETICISTA FRENTE AO PACIENTE PORTADOR DE HIDRADENITE SUPURATIVA}

Trabalho de Conclusão de Curso apresentado como requisito parcial para obtenção do título de Especialização em Procedimentos Estéticos Intradérmicos e cutâneos, pelo Instituto Educacional Wilson Wanderley. Sob a orientação do Prof. Mestre José Francisco Vitarelli 


\section{ESTRATÉGIAS TERAPÊUTICAS DO ESTETICISTA FRENTE AO PACIENTE PORTADOR DE HIDRADENITE SUPURATIVA}

Trabalho de Conclusão de Curso apresentado como requisito parcial para obtenção do título de Especialização em Procedimentos Estéticos Intradérmicos e cutâneos, pelo Instituto Educacional Wilson Wanderley. Sob a orientação do Prof. Mestre José Francisco Vitarelli

Santo André,

Prof. Dr.

Universidade

Prof. Dr.

Universidade

Prof. Dr.

Universidade 


\title{
Estratégias terapêuticas do esteticista frente ao paciente portador de hidradenite supurativa
}

\author{
Lucimara Sargiani Fabossi ${ }^{1}$
}

\section{Resumo}

A Hidradenite Supurativa (HS), também chamada de Hidrosadenite, Acne Inversa ou Doença de Verneuil é uma doença inflamatória crônica, recorrente, estigmatizante e debilitante. Sua etiopatogênese envolve oclusão folicular e fatores genéticos, ambientais e imunológicos, podendo ocorrer isolada ou simultaneamente em diversas regiões do corpo.

As características iniciais da HS são comumente equivocadas com furunculoses, carbúnculos e foliculites, levando a um atraso no diagnóstico e no agravamento das lesões, na necessidade de intensificar a terapêutica medicamentosa e muitas vezes, o afastamento das atividades laborais.

O objetivo deste trabalho é inserir o Esteticista Cosmetólogo em equipe multidisciplinar para o tratamento aos portadores de HS, oferecendo técnicas terapêuticas alternativas complementares às medicamentosas e de controle evolutivo da doença. Para tanto, intensificar a abordagem do tema nos cursos de formação do Técnico em Estética e nas graduações de Esteticista Cosmetólogo.

Por ser uma doença ainda sem cura, e os tratamentos disponíveis são muitas vezes ineficazes, portanto é de fundamental importância novas estratégias terapêuticas que possam contribuir significativamente para o aumento da qualidade de vida dos portadores de HS.

Palavras-chave: Hidradenite Supurativa, Hidrosadenite, Acne Inversa, Doença de Verneuil, Esteticista Cosmetólogo.

\footnotetext{
${ }^{1}$ Graduada em Tecnóloga em Estética e Cosmética - Universidade Braz Cubas - Bragança Paulista (SP). Pós Graduada em Docência para Ensino Superior - Universidade Braz Cubas - Bragança Paulista (SP). Pós Graduando em Procedimentos Intradérmicos e Subcutâneos - Instituto Educacional Wilson Wanderley - Santo André (SP). Contato e-mail: lucimarasargiani@hotmail.com.

Trabalho de Conclusão de Curso apresentado como requisito parcial para obtenção do título de Especialização em Procedimentos Estéticos Intradérmicos e Subcutâneos, pelo Instituto Educacional Wilson Wanderley. Orientador: Prof. Mestre José Francisco Vitarelli. Santo André - SP, 2020.
} 


\begin{abstract}
Hidradenitis Suppurativa (HS), also called by Hydrosadenitis, Acne Inversa or Verneuil's Disease is a chronic, recurrent, stigmatizing and debilitating disease. Its etiopathogenesis involves folicular and genetic occlusion, environmental and immunological factors, which may occur singly or simultaneously in various regions of the body.

The initial characteristics of HS are commonly mistaken for furunculosis, carbuncles and folliculitis, leading to a delay in the diagnosis and worsening of lesions, taking to the need to intensify the drug therapy and often, the departure of labor activities.

The objective of this work is to introduce the Beautician, with a multidisciplinary team, in the treatment of HS patients, offering alternative therapeutic techniques complementary to drugs and disease evolutional control. To this end, intensify the approach of the subject in the training courses of the Technician in Aesthetics and in the graduations of Cosmetologist Beautician.

For being a disease with still no cure, and the available treatments are often ineffective, there is a need for new therapeutic strategies that can contribute significantly to increase the quality of life of HS patients.
\end{abstract}

Keywords: Hidradenitis Suppurativa, Hydrosadenitis, Acne Inversa, Verneuil's Disease, Cosmetologist Beautician. 


\section{Introdução}

Doença da pele de caráter crônico, inflamatório, recorrente, debilitante e estigmatizante, a Hidradenite Supurativa atinge $4 \%$ da população, predominantemente mulheres, no período entre o início da adolescência e os 40 anos. Ocorre em regiões corporais que contém glândulas sudoríparas apócrinas, em uma ou mais regiões simultaneamente. Doença multifatorial e seus fatores de risco são histórico familiar, obesidade, tabagismo, hormonais e uso de medicamentos. As lesões podem se agravar por estresse, calor, suor, roupas apertadas, alimentos pró inflamatórios.

No início a Hidradenite Supurativa pode ser confundida com furúnculos, foliculite, lesões acneicas, mas o diagnóstico tardio leva ao agravamento da doença, ao prejuízo na qualidade de vida dos indivíduos afetados, a depressão.

O Esteticista, profissional conhecedor da fisiopatologia do sistema tegumentar, pode, durante a abordagem e atendimento clínico, identificar lesões de Hidradenite Supurativa e encaminhar o paciente ao médico dermatologista para diagnóstico e conduta, abreviando o tempo de diagnóstico e evitando o agravamento da doença.

Para tanto, o Esteticista deve ser preparado desde a formação profissional sobre a identificação, o encaminhamento e a conduta terapêutica alternativa que vise complementar a farmacológica, a fim de proporcionar saúde e bem estar aos portadores de Hidradenite Supurativa.

É uma proposta de integração do Esteticista à equipe multidisciplinar para o paciente com Hidradenite Supurativa, promovendo a saúde e o bem estar, melhorando a qualidade de vida das pessoas. 


\section{Apresentação clínica}

A HS é caracterizada por lesões profundas e inflamatórias nas regiões axilar, inframamária, esternal, inguinal, genital, interglútea e perineal. Há relatos sobre lesões na face, região mentoniana (2-5-6-7-9-10). Afeta a pele onde há maior quantidade de glândulas apócrinas intertriginosas (9). Ocorre a obstrução do folículo pilossebáceo por acumulação de resíduos celulares, formação de nódulos inflamados e extremamente doloridos, podendo evoluir para abcessos fistulizados e interligados (6) na derme profunda, com formação de cicatrizes. As lesões frequentemente drenam exsudato purulento fétido (9). Com a progressão da doença, ocorre formação de fístulas, comedões, fibrose, contraturas dérmicas e endurecimento da pele (7-9). Algumas literaturas consideram como lesões pré cancerosas (2-3).

Os sintomas iniciam-se entre o período após a puberdade e os 40 anos, sendo mais comum em mulheres do que em homens, com proporção de 3 para 1 (2-6-9-10).

Há relatos de casos com lesões que variam de $1 \mathrm{~cm}$ de diâmetro à $7 \mathrm{~cm}$ de diâmetro com $4 \mathrm{~cm}$ de profundidade, com secreções purulentas, submetidos à cirurgias de esvaziamento glandular (7). 


\section{Etiopatogenia}

Os mecanismos fisiopatológicos responsáveis pela Hidradenite Supurativa ainda não se encontram devidamente explicados. Várias fontes sugerem que seja uma doença multifatorial, cujo evento desencadeante é a oclusão folicular, e os fatores de risco são histórico familiar (40\% dos casos), hormonais, obesidade, tabagismo e uso de fármacos (2-5-10).

O paciente tabagista experimenta uma forma mais agressiva da doença, que pode até desaparecer com a cessação do tabagismo. A nicotina presente no cigarro tem papel na estimulação e disfunção da secreção glandular, além de alterar a quimiotaxia dos neutrófilos (2-5-9).

A obesidade pode agravar a Hidradenite supurativa através do aumento da fricção e do estresse mecânico nas pregas cutâneas, desencadeando uma cascata promotora da oclusão folicular. Adicionalmente, a obesidade caracteriza-se por um estado pró-inflamatório, obtendose um efeito de sinergia com os mecanismos fisiopatológicos de base da HS (2-5-9-10).

Exacerbações podem ocorrer por estresse, calor, suor e roupas apertadas. Pacientes

com HS também podem ser afetados por acne, cistos pilonidais e foliculite crônica do escalpo, condição conhecida como Tétrade da Oclusão Folicular (9).

O método mais utilizado para a classificação da HS é a classificação de Hurley, conforme Tabela $1(10)$.

Tabela 1 - Classificação de Hurley

\begin{tabular}{c|c}
\hline $\begin{array}{c}\text { Estágio I } \\
\text { (ligeiro) }\end{array}$ & Presença de nódulos inflamatórios e abcessos, mas sem fibrose ou cicatrizes \\
\hline $\begin{array}{c}\text { Estágio II } \\
\text { (moderado) }\end{array}$ & $\begin{array}{r}\text { Presença de nódulos inflamatórios e abcessos com fibrose associada e cicatrizes, } \\
\text { embora separadas por regiões íntegras, sem lesões }\end{array}$ \\
\hline $\begin{array}{c}\text { Estágio III } \\
\text { (grave) }\end{array}$ & Envolvimento difuso, com processos fibróticos extensos e múltiplos sinus \\
\hline
\end{tabular}

Fonte: Urmal et al. (2016)

Alguns exemplos dos estágios da Tabela 1, conforme Figuras 1, 2 e 3. 
Figura 1 - Típico caso de paciente com HS no estágio I de Hurley

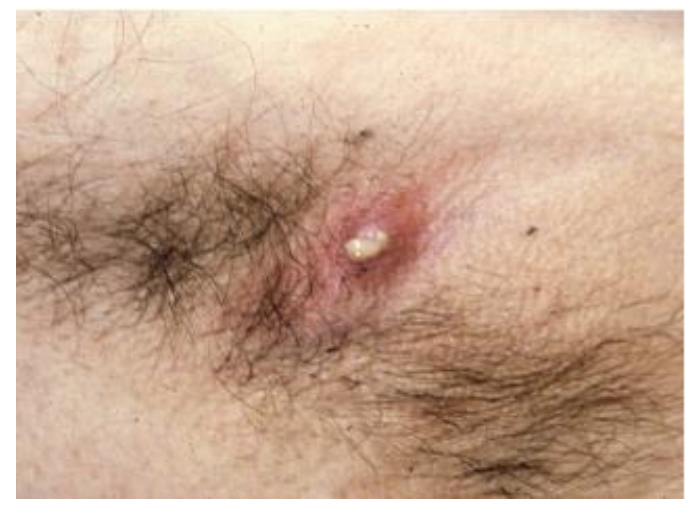

Fonte: Costa-Silva, Azevedo e Lisboa (2018)

Figura 2 - Típico caso de paciente com HS no estágio II de Hurley

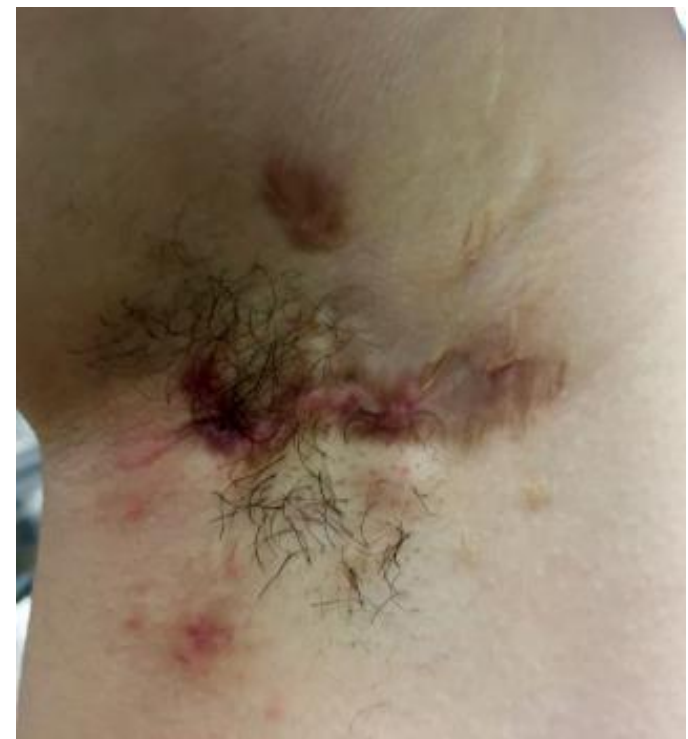

Fonte: Costa-Silva, Azevedo e Lisboa (2018)

Figura 3 - Típico caso de paciente com HS no estágio 3 de Hurley

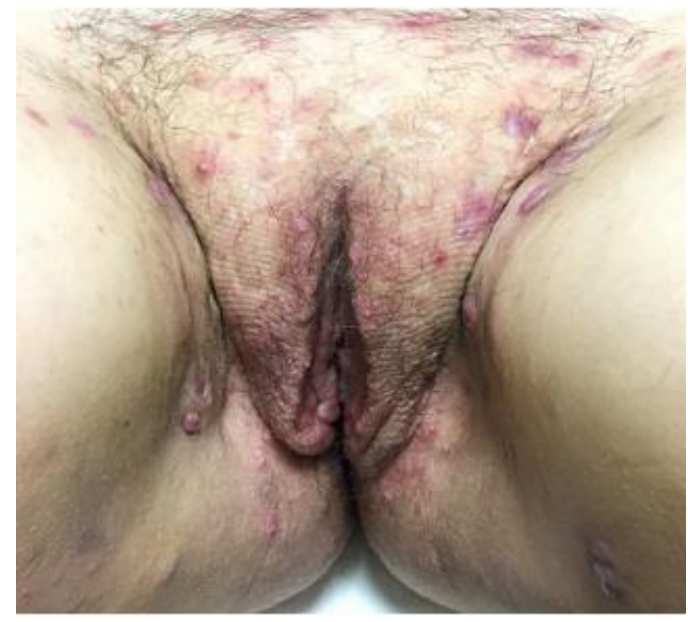

Fonte: Costa-Silva, Azevedo e Lisboa (2018) 


\section{Diagnóstico}

O diagnóstico é predominantemente clínico e a abordagem terapêutica é o principal desafio da doença, devido seu impacto na qualidade de vida. As características muitas vezes inespecíficas da HS levam ao atraso do diagnóstico, tendo o paciente apresentado seu problema para vários especialistas, sem, no entanto, definir um diagnóstico. $\mathrm{O}$ atraso é de 7 anos em média, podendo chegar a décadas. A diferenciação baseia-se na história clínica, aparência, idade de início, localização típica e exame histológico. O diagnóstico diferencial mais comum são foliculites, furunculoses e carbúnculos (9).

Apesar da dor ser a principal característica, o quadro álgido, o mau odor pela saída das secreções e a formação de cicatrizes causam impactos relevantes na qualidade de vida dos pacientes. Embora seja prevalente na população e com opções terapêuticas limitadas, o correto tratamento nesses pacientes é necessário devido à maior presença de depressão e disfunções sexuais em pacientes de HS (6) . 


\section{Tratamentos}

Atualmente, há 3 terapêuticas para o manejo dos pacientes: tratamentos tópicos, sistêmicos e cirúrgicos (6).

Antibióticos tópicos e orais, anti-inflamatórios, isotretinoína, dapsona, metformina, inibidores de TNF-alfa, finasterida, terapias cirúrgicas convencionais, lasers (CO2, Nd:YAG) (2-5-6-9)

Tipos de tratamentos para HS, conforme Tabela 2.

Tabela 2 - Tratamento de Hidradenite Supurativa

\section{TRATAMENTO DE HIDRADENITE SUPPURATIVA DE ACORDO COM A FASE HURLEY}

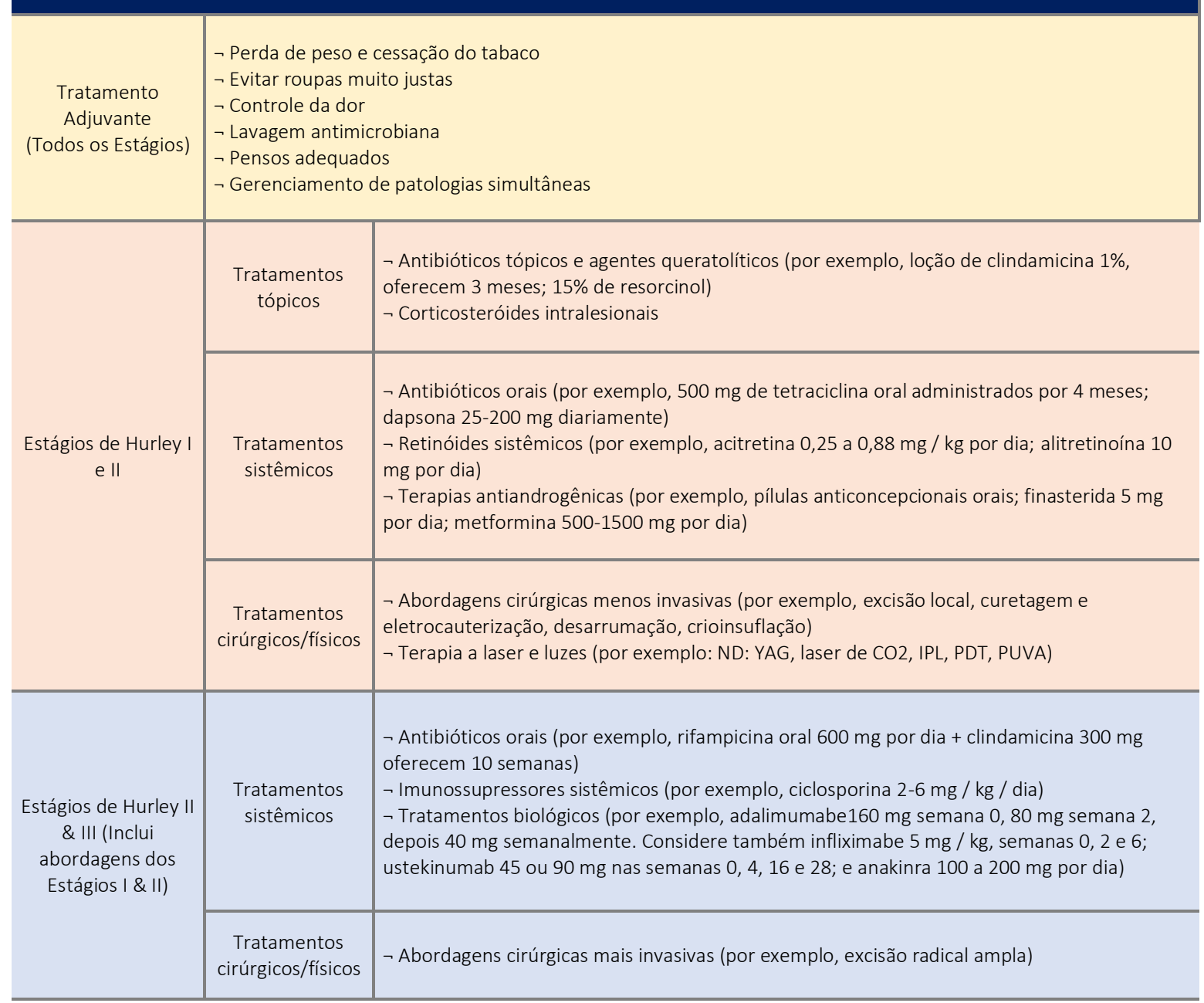

Fonte: Costa-Silva, Azevedo e Lisboa (2018) 
positivo em 2. No entanto, o mecanismo de ação, posologia e duração do tratamento ainda são desconhecidos. Por esse motivo, esse tratamento ainda é considerado experimental (1).

A HS continua a ser uma doença sem cura e cujos tratamentos disponíveis são muitas vezes ineficazes, portanto tem-se a necessidade de novas estratégias terapêuticas que possam contribuir significativamente para o aumento da qualidade de vida dos doentes (5).

A abordagem precoce adequada e multidisciplinar oferece as melhores chances de controle da doença. O tratamento envolve medidas preventivas, clínicas, cirúrgicas e psicológica. Uma vez feito o diagnóstico, deve-se acessar a gravidade e localização das lesões para que as diferentes modalidades de tratamento possam ser usadas de forma individualizada, de acordo com as circunstâncias e preferências do paciente (9). 


\section{O esteticista frente o portador de HS}

Este trabalho sugere que o esteticista cosmetólogo, profissional regulamentado conforme a lei 13.643 de 03 de abril de 2018, preparado durante sua formação acadêmica para atuar no sistema tegumentar, com conhecimentos profundos em anatomofisiologia da pele e seus anexos, que promove além de beleza, a saúde e o bem estar do indivíduo, ofereça técnicas de terapias alternativas, medidas não farmacológicas que visam complementar os tratamentos médicos aos portadores de HS.

Sendo o esteticista cosmetólogo o profissional da área da saúde que oferece tratamentos corporais com finalidades estéticas e de bem estar, como depilação e epilação, massagens, tratamentos para redução de adiposidades e fibroedemagelóide (FEG), limpeza de pele e controle de oleosidade, entre outros, frequentemente recebe possíveis portadores de HS, que procuram esse profissional muitas vezes, queixando-se de "pêlos encravados", "comedões" nas regiões inguinais, interno de coxas e axilares, ou apenas para tratar adiposidades localizadas. Durante a avaliação ou procedimento, o esteticista pode identificar características suspeitas comuns da HS e encaminhar ao médico dermatologista para diagnóstico e conduta. Confirmando a patologia, pode oferecer técnicas terapêuticas alternativas não farmacológicas complementares às médicas, objetivando o controle da evolução dos estágios da doença e no alívio dos sintomas e desconfortos causados por ela.

Nesse sentido, muitos portadores da HS podem ter o diagnóstico correto e o

tratamento específico para cada caso muito antes do agravamento de seus estágios, diminuindo significativamente o tamanho e a quantidade de lesões, a dor, a terapêutica medicamentosa e os agravos provocados por ela, como a depressão, o afastamento das atividades laborais dos pacientes em estágios mais avançados devido à dores, pruridos, edemas, secreções, odores e o desconforto que, muitas vezes, impedem ou dificultam o movimento do doente. $\mathrm{O}$ atraso no diagnóstico da HS leva ao agravamento das lesões. Algumas literaturas consideram as lesões de Hidradenite como pré cancerosas.

O esteticista, no uso de suas competências e habilidades, pode promover o afinamento do estrato córneo, com o objetivo de remover resíduos celulares e o tamponamento dos óstios; realizar a extração dos comedões que se formam nas regiões afetadas, retirando o sebo que se acumula formando nódulos e favorecendo a proliferação de bactérias que provocam a inflamação; promover a descontaminação local através de eletroterapia, com aparelhos de vapor 
de ozônio ou de alta frequência, que promove também a cicatrização; o uso de laser de baixa intensidade, a ledterapia e terapia fotodinâmica (2-4-9), com reconhecidos efeitos antiálgico, anti-inflamatório, regenerativo e circulatório (4), por fotoinibição e fotoestimulação celulares, respeitando as contra indicações relativas e absolutas, principalmente nos pacientes que fazem uso de isotretinoína.

A utilização das técnicas de epilação por aparelhos de laser de fotodestruição dos folículos pilosos (8) e Luz Intensa Pulsada (LIP) (2) também são indicadas aos portadores de HS, respeitando-se o fototipo segundo escala de fitzpatrick, o grau de HS segundo a classificação de Hurley, as contra indicações ao pacientes de isotretinoína e retinóides.

Vários estudos relacionam obesidade com o risco de HS, sendo maior a prevalência da doença em pessoas obesas. Apesar de também estar descrita uma relação entre o aumento do IMC (Índice de Massa Corporal) e a gravidade ou duração da doença, existem também alguns estudos que não corroboram esta relação. A relação entre obesidade e a HS pode ser explicada pela habilidade dos adipócitos produzirem citocinas pró-inflamatórias e pela ação mecânica provocada pelo aumento das pregas cutâneas, que favorece o calor, a humidade e a fricção nas zonas afetadas, contribuindo dessa forma para a manutenção e/ou exacerbação das lesões. Adipócitos maduros secretam adipocinas tais como a TNF-alfa, acelerando processos inflamatórios (1) .

Assim, o paciente de HS deve também ter acompanhamento clínico de nutricionista, para diminuição e controle do IMC através de prescrição de dietas específicas às necessidades individuais, evitando alimentos pró inflamatórios.

Para pacientes com sobrepeso e/ou gordura localizada, o esteticista executa técnicas de ação lipolítica com o emprego de aparelhos eletroterápicos em regiões íntegras, que ainda não apresente lesões, com finalidade preventiva, como ultrassom, radiofrequência, eletrolipólise, carboxiterapia, cavitação, criofrequência, mesoterapia, dentre outros, segundo a fisiopatologia das adiposidades apresentadas (se compacta, se flácida, se edematosa).

Acompanhamento psicológico também é indispensável em alguns casos com maior severidade. A HS tem efeito de longo alcance em todas as áreas da vida. Problemas no ambiente familiar e social, ideias suicidas, medo de estimagtização, dificuldades econômicas contribuem para carga substancial da doença. Alguns estudos destacam a significativa taxa de incapacidade no trabalho justamente com a alta taxa de desemprego entre os portadores de HS. Um contribuinte adicional para a qualidade de vida prejudicada é a disfunção sexual por causa da influência da HS em relacionamentos íntimos e atividade sexual (2). 


\section{CONCLUSÃO}

Os artigos $5^{\circ}$, Incisos II e III e $6^{\circ}$ em sua totalidade da Lei 13.643 de 03 de Abril de 2018, dá-nos essa direção, por completo: solicitar de outro profissional (neste caso, o médico dermatologista) parecer que complemente a avaliação (que teve início com propósitos estéticos e de bem estar); a responsabilidade técnica da execução dos recursos utilizados; o ensino nas disciplinas correspondentes, preparando essa abordagem e terapêutica, elaborando informes e pareceres científicos, elaborando programas de atendimentos individuais, observando e solicitando prescrições e pareceres médicos.

A HS deve ser melhor divulgada entre os profissionais de saúde que podem ser envolvidos em sua terapêutica, e ter uma abordagem diferenciada nos cursos que formam técnicos esteticistas e esteticistas cosmetólogos para que os casos suspeitos sejam encaminhados ao médico dermatologista e o tempo para o correto diagnóstico seja reduzido, evitando assim o agravamento da doença.

Além disso, as técnicas propostas devem ser melhor discutidas, envolvendo profissionais de todas as categorias citadas, tendo as atividades e informações compartilhadas num ambiente de cooperação interdisciplinar, respeitando os limites das diferentes competências e formações profissionais, sem perder de vista a promoção da vida, saúde e bem estar das pessoas.

O diagnóstico correto permite o controle da patologia através de terapias medicamentosas e alternativas, melhorando significativamente a qualidade de vida do indivíduo.

Assim, proponho a discussão e inclusão deste tema na disciplina de dermatologia estética /patologias nos cursos de graduação de esteticista cosmetólogo e na formação do Técnico em Estética. 


\section{REFERÊNCIAS}

1. ALVES, Cláudia Alexandra Rodrigues. Hidradenite Supurativa: O Desafio do Tratamento. Estudo Geral. [s.1.], 2017. Disponível em: 〈http://hdl.handle.net/10316/81970>. Acesso em: 2 Jan. 2020.

2. COSTA-SILVA, M; AZEVEDO, F; LISBOA, C. Avanços na Hidradenite Supurativa: Da Etiopatogenia ao Tratamento. Revista SPDV, 2018. Disponível em: <https://pdfs.semanticscholar.org/e8ca/8f185b6501dc4ce5551675a16e3d3b884bb4.pdf>. Acesso em: 4 Jan. 2020.

3. HIRATA, Sergio Henrique et al. Temas Em Psico-Oncologia . São Paulo: Summus, v. $1,2008$.

4. ISSA, Maria Cláudia Almeida; MANELA-AZULAY, Mônica. Terapia fotodinâmica: revisão da literatura e documentação iconográfica. Anais Brasileiros de Dermatologia, [s.1.], v. 85, n. 4, p. 501-511, ago. 2010. FapUNIFESP (SciELO). http://dx.doi.org/10.1590/s036505962010000400011. Disponível em: <http://www.scielo.br/scielo.php? script=sci_arttext\&pid=S0365-05962010000400011>. Acesso em: 3 Jan. 2020.

5. LOPES, Jéssica Ferreira. Estratégias Terapêuticas na Hidrosadenite Supurativa. Universidade de Coimbra. [s.1.], 2018. Disponível em: <https://estudogeral.sib.uc.pt/handle/10316/84578>. Acesso em: 30 Dez. 2019.

6. MUZY, Guilherme; CROCCO, Elisete Isabel; ALVES, Renata Oliveira. Hidradenite supurativa: atualização e revisão de suas modalidades terapêuticas. Surgical \& Cosmetic Dermatology, [s.1.], v. 6, n. 3, p. 206-211, 2014. Sociedade Brasileira de Dermatologia. Disponível em: <http://www.surgicalcosmetic.org.br/detalhe-artigo/337/Hidradenitesupurativa--atualizacao-e-revisao-de-suas-modalidades-terapeuticas>. Acesso em: 29 Dez. 2019.

7. OBADIA, Daniel Lago. Hidradenite supurativa tratada com infliximabe. Anais Brasileiros de Dermatologia, [s.1.], v. 84, n. 6, p. 695-697, dez. 2009. FapUNIFESP (SciELO). 
http://dx.doi.org/10.1590/s0365-05962009000600022. Disponível em:

<http://www.scielo.br/scielo.php?script=sci_arttext\&pid=S0365-05962009000600022>.

Acesso em: 27 Dez. 2019.

8. OLIVEIRA, M.A.R. et al. Depilação à Laser : Revisão de Literatura. Revista Saúde em Foco .

Revista Saúde em Foco, [s.1.], 2018. ed. 10. Disponível em:

$<$ http://portal.unisepe.com.br/unifia/wp-

content/uploads/sites/10001/2018/07/057_DEPILA\%C3\%87\%C3\%83O_A_LASER_REVIS

\%C3\%83O_DE_LITERATURA.pdf>. Acesso em: 28 Dez. 2019.

9. OLIVEIRA, Milton Paulo de; GAZZALLE, Anajara; NARVAES, Gabriel. Hidradenite supurativa (acne inversa): revisão da literatura e relato de caso sobre o tratamento cirúrgico de lesão pré-esternal. Revista Brasileira de Cirurgia Plástica (RBCP) - Brazilian Journal of Plastic Sugery, [s.1.], v. 30, n. 3, p. 487-494, 2015. GN1 Genesis Network. http://dx.doi.org/10.5935/2177-1235.2015rbcp0184. Disponível em:

<http://www.rbcp.org.br/details/1667/pt-BR>. Acesso em: 5 Jan. 2020.

10. URMAL, I et al. Hidradenite Supurativa: Compreender para Tratar. Gazeta Médica, [s.1.], v. 3, n. 4, 2016. 